\title{
-1" \\ CONSTRUCCIÓN \\ DE PORTAFOLIOS \\ DE INVERSIÓN DESDE \\ LAS FINANZAS DEL \\ COMPORTAMIENTO: \\ UNA REVISIÓN CRÍTICA*
}

\author{
Alejandro José Useche Arévalo**
}

* doi: 10.11144/Javeriana.cao28-51.cpif. Este artículo es producto de la investigación “Finanzas Corporativas del Comportamiento" proyecto desarrollado en la Línea de Investigación en Finanzas de la Escuela de Administración de la Universidad del Rosario desde septiembre de 2014 hasta septiembre de 2016. El artículo se recibió el 09/09/2015 y se aprobó el 15/11/2015. Sugerencia de citación: Useche A., A. J. (2015). Construcción de portafolios de inversión desde las finanzas del comportamiento: una revisión crítica. Cuadernos de Administración, 28 (51), 11-43. http://dx.doi.org/10.11144/Javeriana.cao28-51.cpif

* * Doctor of Business Administration de la Swiss Management Center University de Zug, Suiza, 2012. Profesor principal de carrera, Director de postgrados en Management, Rosario GSB - Graduate School of Business, Universidad del Rosario, Bogotá, Colombia.

Correo electrónico: alejandro.useche@urosario.edu.co 
Construcción de portafolios de inversión desde las finanzas del comportamiento: una revisión crítica

\section{Resumen}

En este artículo se presenta una revisión crítica de las principales teorías para la selección de portafolios desarrolladas a la luz de las Finanzas del Comportamiento, las cuales analizan la influencia de factores emocionales y psicológicos sobre las decisiones financieras. Luego de contrastar el enfoque tradicional de la Teoría Moderna del Portafolio con los modelos Primero la Seguridad, Seguridad-Potencial / Aspiración (SP/A), Portafolio Comportamental y Portafolio Maslowiano se concluye que la asignación óptima del método media-varianza no corresponde con los portafolios hallados bajo supuestos comportamentales. Para futuras investigaciones se aportan elementos conceptuales que permiten determinar los perfiles de los inversionistas, con miras a que las instituciones financieras les brinden una mejor asesoría.

Palabras clave: teoría del portafolio, finanzas del comportamiento, decisiones de inversión.

Clasificación JEL: G110, D140

Investment portfolio elaboration with a behavioral finance approach: A critical review

\section{ABSTRACT}

The aim of this paper is to present a critical review of the most relevant theories for portfolio selection built on the Behavioral Finance approach, in which the influence of emotional and psychological factors over financial decisions is analyzed. The traditional Modern Portfolio Theory approach is contrasted with behavioral models such as Safety First, Security-Potential/Aspiration (SP/A), Behavioral Portfolio, and Maslowian Portfolio, to conclude that the optimal mean-variance allocation does not necessarily correspond to those found under behavioral assumptions. This article provides conceptual elements for a better understanding of the profiles and interests of the investor, as a basis for future applied research oriented to support the investment advice process in financial institutions.

Keywords: Portfolio theory, behavioral finance, investment decisions.

JEL Classification: G110, D140

\section{Construção de portfólios de investimento a partir das finanças comportamentais: uma revisão crítica}

\section{Resumo}

Neste artigo apresenta-se uma revisão crítica das principais teorias para a seleção de portfólios desenvolvidas à luz das Finanças Comportamentais, que analisam a influência de fatores emocionais e psicológicos sobre as decisões financeiras. Após contrastar o enfoque tradicional da Teoria Moderna do Portfólio com os modelos Primeiro a Segurança, Segurança-Potencial/Aspiração (SP/A), Portfólio Comportamental e Portfólio Maslowiano, conclui-se que a designação apropriada do método da média variância não corresponde aos portfólios encontrados sob supostos comportamentais. Para futuras pesquisas, contribuem-se com elementos conceituais que permitem determinar os perfis dos investidores com vistas a que as instituições financeiras lhes ofereçam uma melhor assessoria.

Palavras-chave: teoria do portfólio, finanças comportamentais, decisões de investimento.

Classificação JEL: G110, D140 


\section{Introducción}

La teoría y la práctica financieras han sido profundamente influenciadas por el enfoque tradicional para la selección de portafolios de inversión creado por Harry M. Markowitz (1952) en el artículo clásico Portfolio Selection, en el que formuló el método de media-varianza para la conformación de carteras óptimas, enfoque bajo el cual se supone un agente racional que desea minimizar el riesgo sujeto a un nivel de retorno mínimo esperado, o maximizar la rentabilidad sujeto a un máximo de riesgo deseado (Arcos, Benavides y Breggrun, 2010). También fueron muy influyentes los estudios de William F. Sharpe (1964), John V. Lintner Jr. (1965) y Jan Mossin (1966), quienes basados en los aportes de Markowitz postularon de forma independiente el Modelo de Valoración de Activos Financieros (CAPM), modelo de equilibrio con el cual es posible hallar la tasa de retorno esperada de un activo riesgoso, mediante una ecuación lineal que añade a la tasa libre de riesgo una prima de riesgo, acorde con la sensibilidad del retorno del activo riesgoso frente al comportamiento del mercado, expresada con el coeficiente Beta (Dubova, 2005; González y Nave, 2014) ${ }^{1}$. Estos aportes serían reconocidos en 1990 con el Premio Nobel de Economía, concedido de manera conjunta a Markowitz, Sharpe y Merton “por su trabajo pionero en la teoría de la economía financiera" (Swedish Academy, 2015).

Posteriormente, Fischer Black y Robert Litterman (1991) hicieron una combinación del CAPM y el enfoque media-varianza de Markowitz, en el cual se utiliza un enfoque bayesiano para mezclar las consideraciones particulares de un inversionista sobre el desempeño de varios activos con las condiciones de equilibrio del mercado, para encontrar portafolios intuitivos y diversificados, modelo que recibe el nombre de Black-Litterman.

Este conjunto de desarrollos es conocido como Teoría Moderna del Portafolio, la que al día de hoy tiene el reconocimiento de ser "el fundamento principal de la teoría financiera que es enseñada alrededor del mundo" (Auðunsdóttir, 2011, p. 5). Sin embargo, a pesar de su rigor en términos de la formalización cuantitativa, ella se basa en supuestos que pueden ser criticables como, por ejemplo, que los agentes descritos están siempre en capacidad de tomar decisiones óptimas, acordes con sus preferencias constantes frente al riesgo, con base en criterios objetivos y de total racionalidad, en el marco de mercados financieros eficientes (Frijns et al., 2008; Schulmerich et al., 2015).

1 El crédito por la creación del CAPM también corresponde a Jack Treynor, quien precedió a Sharpe, Lintner y Mossin con su trabajo "Toward a Theory of Market Value of Risky Assets", documento que nunca fue publicado. 
Avances posteriores - menos difundidos o incluso ignorados en la literatura tradicionalproponen enfoques alternativos que toman en cuenta aspectos propios de la condición humana, como emociones, factores psicológicos, influencia del entorno y subjetividad en los juicios, que claramente inciden en la toma de decisiones financieras, surgiendo así una escuela de pensamiento denominada Finanzas del Comportamiento. En este sentido, el objetivo del presente artículo es presentar una revisión crítica de las principales teorías para la selección de portafolios de inversión que se han desarrollado a la luz del enfoque comportamental, como complemento y a la vez alternativa a la tradición ortodoxa descrita por la Teoría Moderna del Portafolio². La exposición temática se hace en este orden: primero se hace una descripción de las principales variables psicológicas que influyen en la selección y gestión de portafolios óptimos de inversión. En la segunda parte se presenta una descripción de las diferencias entre la teoría tradicional y el enfoque comportamental para la selección de carteras de inversión. En la tercera parte se analizan la teoría Primero la Seguridad, de Roy, el enfoque Seguridad-Potencial / Aspiración (SP/A) de Lopes y Oden, la Teoría Comportamental del Portafolio de Shefrin y Statman, en sus dos modalidades (cuenta única y cuentas múltiples), y la Teoría Maslowiana del Portafolio desarrollada por De Brower. Finalmente, en la cuarta parte se presentan las conclusiones y se dan recomendaciones para futuras investigaciones.

\section{Aspectos psicológicos que influyen en la selección y gestión de portafolios de inversión}

Las Finanzas del Comportamiento se han desarrollado en gran medida por sus fundamentos en aspectos psicológicos aplicados al estudio del comportamiento de los inversionistas. De acuerdo con Pascual-Ezama, Gil-Gómez de Liaño y Scandroglio (2015), la psicología social ha desarrollado modelos para estudiar el comportamiento humano, dentro de los que resaltan la Teoría de la acción razonada (Fishbein y Ajzen, 1975), el Modelo de motivación y oportunidad como determinantes de procesamiento (Fazio, 1986), y la Teoría del comportamiento planificado (Ajzen, 1991), el cual es considerado como el más satisfactorio para estudiar el comportamiento humano en términos de las decisiones económicos y de inversión (Cooke y Sheeran, 2004).

2 La pertinencia de la literatura presentada fue corroborada por medio de un análisis cienciométrico realizado con el software Harzing's Publish or Perish @ , con el cual se revisó el impacto de las publicaciones en el campo mediante el Índice $h$, el cual se basa en la cantidad de citas que recibe un determinado artículo científico: un autor recibe un valor $h$ si ha publicado $h$ trabajos, cada uno con al menos $h$ cantidad de citas. 
Aunque la literatura al respecto es extensa, vale la pena resaltar los trabajos de compilación realizados por Shefrin (2007), Pascual-Ezama, Pavonni y Gil-Gómez de Liaño (2010), Nofsinger (2012), Baker y Ricciardi (2014), y Richards (2014). Se presentan a continuación los principales aspectos psicológicos a los que se refieren las Finanzas del Comportamiento cuando intentan explicar cómo las emociones afectan el proceso de toma de decisión, y la forma en que las personas construyen sus creencias.

\subsection{Las emociones y el entorno}

- Estados de ánimo (mood): la psicología afirma que los estados emocionales influencian profundamente las decisiones de las personas: un buen estado de ánimo tiende a hacer que una persona se sienta más optimista en sus inversiones y, por lo tanto, más dispuesta a tomar riesgos (Johnson y Tversky, 1983; Wright y Bower, 1992); aún más, cuando hay optimismo en el ambiente, los análisis de inversión se llevan a cabo de forma menos crítica y detallada (Nofsinger, 2002). Por el contrario, un mal estado de ánimo lleva a que un inversionista evalúe más detalladamente la información y lo haga con algo de pesimismo, sea menos proclive al riesgo y conforme su portafolio con títulos que considere los más seguros (Forgas, 1995; Loewenstein et al., 2001; Dowling y Lucey, 2005). Diversos estudios han encontrado unas altas correlaciones entre las tasas de retorno de los mercados accionarios y factores como el clima, las fases lunares y los resultados deportivos, situaciones que afectan el ánimo y, a su vez, este influye en las decisiones de inversión (Eagles, 1994; Kamstra, Kramer, y Levi, 2003; Yuan, Zheng y Zhu, 2006; Edmans, García y Norli, 2007). Las emociones positivas llevan a que los inversionistas eviten incorporar datos que puedan contradecir sus predicciones, minimizando la importancia de información que entre en conflicto con sus creencias y, en lugar de esto, busquen evidencia a favor de sus ideas arraigadas, conducta que recibe el nombre de "disonancia cognitiva" (Akerlof y Dickens, 1982; Barberis y Thaler, 2003; Chandra, 2008; Kuhnen y Knutson, 2011).

- Arrepentimiento (regret): esta es una reacción psicológica que se produce ante una decisión incorrecta, o cuando se rechaza una alternativa que luego resulta ser la acertada (Bell, 1982; Nofsinger, 2001). La sensación de arrepentimiento tiende a ser mayor cuando se toma una decisión excepcional, que se sale de los patrones tradicionales: por ejemplo, si un inversionista históricamente ha adquirido títulos de renta fija, pero en determinado periodo decide invertir en títulos de renta variable, los cuales bajan luego de precio y le generan pérdidas (Shefrin, 2007); 
inversionistas que tengan temor al arrepentimiento tenderán a conservar sus estrategias tradicionales y a aplicar métodos pasivos de gestión de portafolios.

- Miedo y codicia (fear, greed): el miedo es una sensación que se genera por la posibilidad de sufrir una pérdida, que lleva a evitar decisiones riesgosas, de forma que un inversionista que siente miedo tiende a adquirir activos de renta fija, menos volátiles, que le permitan garantizar un nivel de consumo deseado (Shefrin y Statman, 2000). Por otra parte, la codicia se relaciona con el ánimo de obtener ganancias a partir de la adquisición de activos riesgosos: los inversionistas "codiciosos" adquieren acciones y activos más volátiles, sobre los que hay más incertidumbre, estructurando portafolios poco diversificados (Statman, 2002). De esta manera, como lo demostraron Mayfield, Perdue, y Wooten (2008), la intención de inversión varía en función del tipo de personalidad.

- Comportamiento de manada (herd behavior): tendencia de los individuos a seguir el comportamiento observado de los grupos. En los mercados financieros, este efecto se presenta cuando las decisiones de compra o venta de un activo se ven influenciadas por comentarios de otros - a veces irrelevantes-y por creencias populares (Shiller, 1990; Hirshleifer, 2001), llevando a que diferentes inversionistas sigan a un grupo de líderes en sus decisiones de portafolio, sin realizar análisis detallados sobre riesgos, rentabilidad o correlación (Nofsinger y Sias, 1999; Sias, 2004). Las burbujas en los mercados de valores y las innovaciones financieras en las que los inversionistas quedan atrapados en un estado emotivo en el que inconscientemente subestiman el riesgo de tales nuevos productos, pueden ser consideradas ejemplos del comportamiento de manada (Taffler y Tuckett, 2010). Al respecto, Fan, Ying, Wang y Wei (2009) afirman que los factores psicológicos tienen profundos impactos en el desempeño macroeconómico y pueden generar graves crisis financieras, argumento que es desarrollado por medio de un modelo de inteligencia artificial basado en "células autómatas" que tratan de replicar la complejidad del mercado de capitales.

\subsection{Formación de creencias}

La psicología afirma que la mente humana cuenta con una habilidad limitada de concentración y de procesamiento de la información, incluso que las decisiones se ven afectadas significativamente por el subconsciente (Szyszka, 2013). Dentro de los fenómenos psicológicos más destacados que afectan la manera en que se percibe y se procesa la información financiera se encuentran: 
- Efecto de encuadre (framing effect): llevar a cabo análisis de un problema sin considerar suficientemente bien su entorno, o de una manera muy aislada. Kahneman y Tversky $(1979,1981,1986)$ encontraron que, dada una percepción limitada de una situación, las personas suelen mostrar preferencias cambiantes y tomar decisiones radicalmente distintas cuando se enfrentan a un mismo problema que es presentado de formas diferentes. Así, las decisiones de inversión dependen de la forma en que se presentan las alternativas: por ejemplo, dado que las personas suelen ser más sensibles a las pérdidas que a las ganancias, una opción que resalte la posibilidad de ganancias asociadas a una decisión la hace más atractiva que otra en que se mencione la posibilidad de pérdidas (Rabin, 1998).

- Contabilidad mental (mental accounting): proceso por el cual el cerebro tiende a definir metas y actuar hacia ellas de forma separada unas de otras, lo cual ayuda a medir el progreso particular de cada una (Thaler, 1980, 1985, 1990, 1999). Como se explicará más adelante, Shefrin y Statman (2000) aplican este concepto a la teoría de selección de portafolios afirmando que el inversionista considera el retorno esperado y el riesgo de cada activo como los elementos fundamentales para la toma de decisiones, pero, dada la contabilidad mental, en la práctica suele pasar por alto la interacción entre los activos, construyendo su portafolio a manera de una pirámide escalonada en la que cada segmento representa una meta particular de inversión independiente de los demás segmentos. Tal comportamiento está relacionado con el "encuadre limitado" (narrow framing), que consiste en la propensión de algunos inversionistas a seleccionar los activos de forma individual, pasando por alto el impacto sobre el portafolio en conjunto.

- Disponibilidad (availability): cuando las personas juzgan la probabilidad de un suceso, por lo general buscan información al respecto en sus memorias, bien sea de experiencias propias o de datos obtenidos a través de terceros; aunque este es un proceso lógico, puede generar estimaciones de probabilidad sesgadas pues dependen de la información que esté disponible en la mente de cada uno o de la capacidad de recordarla y analizarla (Tversky y Kahneman, 1974). En particular, las personas tienden a hacer juicios basadas en eventos recientes, fáciles de traer a la memoria, o en sucesos que tuvieron importantes efectos emocionales en el pasado reciente, por encima de hechos más relevantes pero difíciles de recordar (Chandra, 2008). Este efecto lleva a minimizar la importancia del tamaño de muestra considerada, es decir, a juzgar la probabilidad de un suceso con base en muestras muy pequeñas, aquellas que están frescas en la memoria. Así, cada inversionista toma sus decisiones de portafolio mediante análisis subjetivos de 
probabilidades, en un proceso sesgado en el que define eventos, probabilidades y riesgos de acuerdo con sus propias experiencias recientes.

- Exceso de confianza (overconfidence): la gente suele pensar que sus habilidades para ciertas actividades son mayores que las de la persona promedio: basta con preguntar a un grupo de personas cuántas de ellas piensan que conducen automóvil mejor que el conductor común (Stael von Holstein, 1972). De la misma forma, en los mercados financieros muchos inversionistas piensan que sus conocimientos $y$ habilidades son sobresalientes, principalmente en lo relacionado con la selección de activos y las proyecciones sobre sus comportamientos (Barber y Odean, 2001). Se desprenden de aquí varias implicaciones para la determinación de portafolios: por un lado, la ilusión de conocimiento (Baker y Nofsinger, 2002) lleva a que la mayor disponibilidad de información genera la sensación de un mayor conocimiento del mercado; por otra parte, el exceso de transacciones, pues el inversionista que presenta esta característica compra y vende activos rápidamente, pero sus tasas de rentabilidad son en promedio menores que las obtenidas por aquellos que mantienen inalterado su portafolio, debido, entre otros factores, a los costos de transacción como comisiones e impuestos (Barber y Odean, 2000, 2001; Beyery Bowden, 1997).

- Exceso de optimismo (excessive optimism): tendencia psicológica por la cual los agentes sobreestiman la posibilidad de disfrutar de escenarios futuros positivos y minimizan la posibilidad de afrontar situaciones negativas (Baker y Wurgler, 2013). Los inversionistas optimistas tienden a pronosticar mayores rentabilidades de los activos, entornos más adecuados, mejor comportamiento de los indicadores macroeconómicos (0lsen, 1997), lo que los lleva a elegir activos más riesgosos, pues subestiman los peligros que ellos podrían representar a futuro. Incluso, el exceso de optimismo lleva al "sesgo de atribución selectiva" (selective-attribution bias), por el cual los inversionistas atribuyen las ganancias obtenidas a sus propias capacidades, mientras culpan a la mala suerte de las pérdidas que sufren (Fischhoff, 1982; Langer y Roth, 1975; Miller y Ross, 1975; Taylor y Brown, 1988).

- Representatividad (representativeness): juicio basado en estereotipos, supuesto de que las cosas que comparten ciertas cualidades son similares. El cerebro tiende a realizar juicios de forma rápida, sustituyendo cálculos de probabilidades por juicios de representatividad: en términos de las inversiones, tal característica lleva a que, por ejemplo, los inversionistas en la bolsa de valores compren acciones que representen cualidades deseables para ellos, confundiendo una buena empresa con una buena inversión, pues una adecuada trayectoria histórica de ganancias y dividendos no implica un mismo comportamiento a futuro (Baker y Nofsinger, 2002; De Bondt y Thaler, 1985; Lakonishok, Shleifer y Vishny, 1994). 
Las Finanzas del Comportamiento son resultado del reconocimiento de la influencia que variables psicológicas, como las que se acaban de describir, tienen sobre las decisiones financieras. Gracias a la conjugación de las finanzas neoclásicas con elementos de la psicología y la sociología se han desarrollado modelos de selección de carteras de inversión que, conservando la formalización cuantitativa, analizan la forma en que elementos subjetivos como las emociones, los sesgos (predisposición sistemática al error) y los heurísticos (simplificaciones mentales inconscientes para toma rápida de decisiones) afectan -positiva o negativamente- las decisiones de adquisición de activos financieros y su gestión a través del tiempo (Ben Mohamed y Abdelfatteh, 2012; Nawrocki y Viole, 2014; Shefrin, 2007; Statman, 2014). En el siguiente apartado se presenta una descripción de los principios de las Finanzas del Comportamiento, comparándolos con los supuestos de la Teoría Moderna del Portafolio, para luego analizar los modelos más representativos de selección de carteras de inversión que se han desarrollado a la luz de principios comportamentales.

\section{Enfoque comportamental para la selección de portafolios}

El modelo media-varianza para la selección de portafolios de inversión tiene una profunda influencia de la tradición microeconómica neoclásica, en la cual se analiza a un agente optimizador, cuya finalidad es optimizar una función objetivo, sujeto a una o varias restricciones. En el caso financiero, Markowitz planteó un proceso en el cual un inversionista desea hallar el porcentaje de fondos que debe asignar a cada uno de varios activos con el fin de obtener un portafolio que maximice la rentabilidad esperada (media), sujeto a la restricción de no exceder una volatilidad máxima deseada (varianza). Claramente este método es una aplicación de la teoría de la utilidad subjetiva esperada, desarrollada por John von Neumann, Oscar Morgenstern y Frank Ramsey, en la cual no se requiere analizar qué factores determinan sus elecciones, ni tener en cuenta aspectos emocionales o psicológicos, ya que los agentes "revelan" sus preferencias a través de sus comportamientos maximizadores, en un escenario de perfecta disponibilidad y procesamiento de la información en el que "realizan cálculos que involucran medidas de utilidad y probabilidades, por medio de los cuales llegan a determinar, libres de errores cognitivos, el grado de satisfacción de cada acto considerado dentro del conjunto de opciones disponibles para elegir el que optimice sus resultados esperados" (Bishop, 2007, pp. 219-220).

La teoría neoclásica de la elección bajo incertidumbre clasificó en tres categorías los perfiles de aversión al riesgo de los inversionistas, a saber: aversos al riesgo (agentes 
que prefieren obtener con certeza el valor esperado de un juego en lugar de jugarlo, generando una función de utilidad cóncava), propensos al riesgo (quienes prefieren jugar y obtienen satisfacción de enfrentarse a la incertidumbre, caracterizado por una función de utilidad convexa) y neutrales al riesgo (indiferentes a jugar o no, lo que resulta en una función de utilidad lineal) (Kreps, 2012; Mas-Colell, Whinston y Green, 1995). Esta tipología no sólo ha sido la base para el desarrollo de modelos teóricos de consumo e inversión, sino también ha servido como guía para la asesoría financiera tradicional brindada por instituciones como compañías de inversión o fondos de pensiones, que en muchas ocasiones se limitan a recomendar activos arriesgados (acciones, divisas) para clientes con perfiles "agresivos", y activos más seguros (certificados de depósito a término, bonos estatales) para clientes "conservadores". Como se describirá a continuación, las Finanzas del Comportamiento aportan elementos para una comprensión más realista de los perfiles de riesgo de las personas, mediante modelos que incluyen diversidad de objetivos e intereses, así como emociones y aspectos psicológicos cuya inclusión puede llevar a mejorar la efectividad de la asesoría a los usuarios de las instituciones financieras.

Como respuesta a las críticas planteadas al enfoque tradicional, varios autores han desarrollado modelos alternativos en los que incluyen aspectos propios del comportamiento humano observado: tal fue el caso del artículo pionero Safety first and the holding of assets, publicado por Arthur D. Roy (1952), en el que criticó el supuesto de la total racionalidad, planteando que emociones como el miedo a una pérdida catastrófica de la riqueza son fundamentales en la decisión de adquisición de los activos financieros que componen una cartera.

En este mismo orden de ideas, es de resaltar el aporte realizado por Herbert Simon, quien fue un fuerte crítico de los modelos ortodoxos abstractos de toma de decisiones, pues consideraba que ellos no explicaban la conducta real de las personas; como alternativa, creó el concepto de "racionalidad limitada", según el cual los seres humanos, dadas sus limitaciones cognitivas, no necesariamente llevan a cabo procesos de optimización, sino que forman un criterio o "aspiración" de qué tan buena alternativa deberían encontrar, de manera que cuando encuentran una opción que cumpla con tal nivel de aspiración, la seleccionan y terminan la búsqueda, proceso en el que son guiados por parámetros personales subjetivos. En sus propias palabras:

Los seres humanos (y otras criaturas) no se comportan óptimamente dadas sus aptitudes, porque son completamente incapaces de adquirir el conocimiento y de hacer cálculos que sustentarían la optimización. No conocen todas las alternativas 
disponibles para actuar; tienen solamente conocimiento incompleto e incierto sobre las variables del entorno, presente y futuro, que determinarán las consecuencias de sus decisiones; y serían incapaces de hacer los cálculos requeridos para una óptima selección incluso si tuvieran conocimiento perfecto... Simplemente lo tomaré como un axioma: los seres humanos son capaces solamente de una racionalidad muy aproximada y limitada (Simon, 1993, p. 156).

Más adelante se presentó un avance trascendental en el desarrollo de enfoques complementarios para la comprensión del proceso de toma de decisiones financieras, iniciado por Daniel Kahneman y Amos Tversky con el artículo el artículo Prospect theory: An analysis of decision under risk (1979), en el que presentaron formalmente la denominada Teoría de la prospectiva, trabajo pionero en la aplicación del enfoque comportamental a la comprensión de las decisiones bajo incertidumbre 3 . Una nueva versión de esta teoría fue presentada por los mismos autores en el documento Advances in prospect theory: Cumulative representation of uncertainty (1992), en el que incluyeron adicionalmente factores de tipo comportamental como aversión a las pérdidas, tendencia a la búsqueda del riesgo, efectos de encuadre, dependencia de la fuente y preferencias no lineales. Vale la pena anotar que la trascendencia de estos aportes ha sido reconocida, al punto de recibir el Premio Nobel de Economía en el año 2002, otorgado conjuntamente a Daniel Kahneman y Vernon Smith, quienes sentaron las bases del enfoque comportamental y la metodología experimental en ciencias económicas, respectivamente.

Pero fue Richard Thaler quien orientó hacia las finanzas el marco conceptual construido por Kahneman y Tversky, por lo que es conocido como el padre de las Finanzas del Comportamiento; entre sus aportes se encuentran la identificación de una serie de sesgos comportamentales (contabilidad mental, efecto dotación) que hacen que las decisiones financieras reales disten de forma sistemática de las descritas en los modelos neoclásicos (Russelly Thaler, 1985) y el desarrollo de modelos en los que no hacen uso de los supuestos tradicionales de preferencias constantes, expectativas racionales y análisis perfecto de la información, tratando de explicar el comportamiento observado del homo sapiens, en lugar de describir cómo debería comportarse el homo oeconomicus (Jolls, Sunstein y Thaler, 1998; Thaler, 2000).

3 Este es el trabajo con mayor Índice $h$ en el área de las Finanzas del Comportamiento, arrojando un valor de 39.405 al momento de elaborar el presente estudio. 
Además de Thaler, varios autores han contribuido al desarrollo del enfoque comportamental en finanzas, como Robert Shiller, Meir Statman, Hersh Shefrin y Nicholas Barberis, entre otros. Incluso autores como Curtis (2004), Lo (2005) y Brennan y Lo (2011) han realizado contribuciones para el desarrollo de un enfoque que reconcilie los puntos de vista tradicional y comportamental en las finanzas, en los que se resalta la importancia de la asesoría financiera para que -en el marco de mercados ineficientes- las firmas de inversión puedan dar consejos realmente eficientes que lleven a que sus clientes puedan cumplir con sus metas particulares y a evitar errores generados por sesgos psicológicos o por el uso de heurísticos en sus procesos de decisión.

Los principios en los que se basan las Finanzas del Comportamiento, a diferencia de las tradicionales o neoclásicas, para la selección de carteras de inversión pueden ser resumidos así:

1. Los agentes toman decisiones financieras con base en criterios objetivos (precios, retornos esperados, impuestos), pero también son influenciados por factores subjetivos como emociones y sesgos psicológicos (comportamiento de manada, juicios por estereotipos, creencias arraigadas, desconfianza en lo desconocido, apego a activos con retornos negativos, entre otros), llevando al uso de heurísticos en sus decisiones.

2. El acceso a la información y principalmente la capacidad de analizarla, son limitados, de manera que el inversionista no es un maximizador hiper-racional, sino una persona con racionalidad limitada -en el sentido propuesto por Herbert Simon.

3. El inversionista tiene como criterio de elección la satisfacción de sus objetivos, más que la maximización en el sentido estricto, de forma que sus decisiones podrían ser subóptimas a la luz de la teoría tradicional.

4. Las personas tienden a construir portafolios por medio de diferentes cuentas mentales, es decir, por segmentos separados, cada una orientada a un fin diferente, pasando por alto la correlación entre cada una de ellas.

5. Los agentes tienden a ser aversos a las pérdidas, de forma que una pérdida de un monto determinado produce un malestar mayor que el bienestar generado por una ganancia del mismo monto.

6. Los mercados de activos financieros no son necesariamente eficientes, de forma que sus precios de mercado no siempre corresponden con sus valores intrínsecos, generando posibilidades de arbitraje y ganancias extraordinarias. 
A continuación se analizarán cuatro teorías que son de especial relevancia para comprender el enfoque comportamental de la selección de portafolios: Safety First (Roy), SP/A (Lopes y Oden), Behavioral Portfolio Theory (Shefrin y Statman) y Maslowian Portfolio Theory (De Brower).

\subsection{Portafolio Primero la seguridad (Safety first)}

En el trabajo de 1952 Safety first and the holding of assets, Arthur D. Roy postuló el principio comportamental de "Primero la seguridad", el cual afirma que la riqueza de las personas se encuentra constantemente expuesta a situaciones externas adversas, por lo cual es lógico que ellas, influidas por el miedo y la necesidad de seguridad, traten de reducir la probabilidad de ocurrencia de una catástrofe financiera. En este enfoque se encuentra una crítica al supuesto tradicional -e inadvertido- de la "supervivencia" de los inversionistas, pues, por lo general, los modelos no toman en cuenta la posibilidad de que ocurra un desastre y la riqueza se pierda parcial o totalmente, eventos denominados “cisnes negros" por Taleb (2010).

En el modelo media-varianza de Markowitz, el inversionista elige una combinación de activos tal que satisfaga sus preferencias en cuanto a rentabilidad $(\mu)$ y riesgo $(\sigma)$ esperados, de forma que maximiza $\mu$ sujeto a un nivel máximo deseado de $\sigma$, o minimiza $\sigma$ sujeto a un nivel mínimo requerido de $\mu$. En términos generales, su objetivo es maximizar su función de utilidad esperada, dada por:

$$
E(U)=U(\mu, \sigma)
$$

Siguiendo a Fairchild et al. (2014) y a Haley et al. (2013), se define una curva de indiferencia que representa la anterior función de utilidad esperada, con un parámetro de aversión al riesgo $\psi$, y con un nivel de utilidad constante $\bar{U}$ para cada curva, dado por:

$$
E(U)=\bar{U}=\mu-\psi \sigma^{2}
$$

Despejando, $\mu=\bar{U}+\psi \sigma^{2}$, lo que muestra relación directa entre el riesgo ( $\sigma$ ) y el retorno esperado $(\mu)$, llevando a que la curva de indiferencia correspondiente tenga pendiente positiva. Como es tradicional, la situación óptima se encuentra en el punto de tangencia de la restricción presupuestaria (representada en este caso por la línea del mercado de 
capitales) con la más alta curva de utilidad que sea posible, todo esto acorde con el modelo original de Markowitz. Ahora bien, bajo el enfoque "Primero la Seguridad", el inversionista define un nivel catastrófico $\hat{c}$, de forma que su función de utilidad es en este caso:

$$
E(U)=\bar{U}=(\mu-\hat{c}) / \sigma>0
$$

De la anterior expresión se puede obtener la curva de indiferencia correspondiente, que viene dada por $\mu=\hat{c}+\sigma \bar{U}$, que resulta ser lineal y también con pendiente positiva. El inversionista que desea evitar la situación catastrófica maximiza entonces $(\mu-\hat{c}) / \sigma$; sin embargo, en este caso no es posible encontrar el punto de tangencia entre la curva de indiferencia y la línea del mercado de capitales, sino que hay muchas posibilidades entre dos situaciones extremas: sabiendo que $\mu_{f}$ es la tasa libre de riesgo, 1) si $\hat{c}<\mu_{f^{\prime}}$ el inversionista asigna la totalidad de sus recursos al activo libre de riesgo, que al tener $\sigma=0$ genera una utilidad que tiende a infinito; 2) si $\hat{c}>\mu_{f^{\prime}}$ el inversionista puede tomar préstamos a la tasa libre de riesgo e invertir montos ilimitados en el portafolio riesgoso. Si $\hat{c}=\mu_{f^{\prime}}$ la solución es indeterminada; al respecto, ver Fairchild et al. (2014) y Pyle y Turnovsky (1970).

Finalmente, el inversionista puede tomar diversas decisiones, cada una con una rentabilidad esperada $\mu$, con un error de predicción $\sigma$, teniendo como finalidad evitar el nivel catastrófico $\hat{c}$; la rentabilidad que realmente se obtenga es una variable aleatoria. Considerando todos los posibles valores que $\mu$ y $\sigma$ pueden asumir, la expresión de la línea envolvente de todos ellos es $f(\mu, \sigma)=0$; Roy planteó que, ya que es imposible calcular con estos datos la probabilidad de $\Omega$, la mejor alternativa es recurrir a la desigualdad de Bienaymé-Tchebycheff para hallar su límite superior:

$$
P(|\Omega-\mu| \geq \mu-\hat{c}) \leq \sigma^{2} /(\mu-\hat{c})^{2}
$$

Para minimizar la probabilidad de entrar en un estado catastrófico, el inversionista debe minimizar $\mathrm{P}(\Omega \leq \hat{\mathrm{c}})$; que es igual que maximizar $(\mu-\hat{\mathrm{c}}) / \sigma$. Si $\sigma$ fuera constante para cualquier valor de $\mu$, sería posible maximizar el retorno esperado maximizando $(\mu-\hat{c}) / \sigma$.

En su trabajo original, Roy planteó su modelo de "Primero la Seguridad" a la asignación de un monto $k$ de dinero entre $n$ activos, cada uno en una proporción $x_{i}$. Suponiendo que los valores de mercado iniciales de los activos son todos de $\$ 1$, sus valores al cabo de 
un periodo son $p_{1^{\prime}}, \ldots, p_{n^{\prime}}$ sus errores estándar $\alpha_{1^{\prime}} \ldots, \alpha_{n^{\prime}}$ y sus correlaciones $\left(r_{12} ; \ldots\right)$, la rentabilidad del portafolio y su varianza vienen dadas respectivamente por:

$$
\begin{gathered}
\Omega=\sum_{i=1}^{n} x_{i} p_{i} \\
\sigma^{2}=\sum_{i=1}^{n} \sum_{j=1}^{n} x_{i} x_{j} r_{i j} \alpha_{i} \alpha_{j}
\end{gathered}
$$

Aunque estas ecuaciones permiten describir el total de la familia de curvas $\mu, \sigma$, es suficiente con conocer su envolvente; para este fin, $a$ es el vector columna $\left(p_{1} / \alpha_{1}, \ldots, p_{n} / \alpha_{n}\right)$, $b$ es el vector columna $\left(1 / \alpha_{1}, \ldots, 1 / \alpha_{n}\right), W$ es la matriz de correlaciones y $W^{-1}$ su inversa, de tal forma que la envolvente se expresa como:

$$
\left[\frac{\left(a^{\prime} W^{-1} a\right)\left(b^{\prime w-1} b\right)-\left(a^{\prime} W^{-1} b\right)^{2}}{b^{\prime} W^{-1} b}\right]\left(\sigma^{2}-\frac{k^{2}}{b^{\prime} W^{-1} b}\right)=\left(\mu-k \frac{a^{\prime} W^{-1} b}{b^{\prime} W^{-1} b}\right)^{2}
$$

Finalmente, el conjunto de valores óptimos de $x_{i}$, entendido como aquel que minimiza la posibilidad de un suceso catastrófico sobre el valor del portafolio, puede ser encontrado así:

$$
x_{i}=\frac{\frac{k}{\left[a-\left(\frac{d}{k}\right) b\right]^{\prime} W^{-1} b}}{\alpha_{i}} \sum_{j=1}^{n} \frac{\left(p_{j}-\frac{d}{k}\right)}{\alpha_{j}} \frac{W_{i j}}{|W|}
$$

en donde la razón d/k es llamada "precio crítico", es decir, un nivel por debajo del que se presenta la situación de desastre, de manera que el inversionista puede conservar los activos cuyos precios sean superiores al crítico, pero sería recomendable vender los que tengan precios inferiores.

Una revisión del modelo de Roy permite ver que hace una crítica importante al supuesto tradicional de los agentes racionales optimizadores (quienes tienen conocimiento preciso de todos los resultados posibles y de sus probabilidades), resaltando que los procesos de toma de decisiones no son necesariamente orientados a maximizar cierta función 
como sí a evitar una pérdida desastrosa. Este enfoque no toma la "supervivencia" como un hecho, aportando la consideración de condiciones propias de la naturaleza humana como el miedo y la ansiedad ante posibles escenarios catastróficos, y la manera en que tales emociones influencian la composición de la cartera que definirá el inversionista con el fin de evitar un desastre potencial.

\subsection{Portafolio Seguridad - Potencial / Aspiración} (security-potential/aspiration - SP/A)

En el artículo Between hope and fear: The psychology of risk (1987), Lola Lopes planteó un marco conceptual desde la psicología para analizar el comportamiento de un inversionista ante una decisión riesgosa, influenciado por el miedo y la esperanza, en el cual destacan dos criterios lógica y psicológicamente separados: por un lado, el temor de no contar con un nivel mínimo de riqueza (Seguridad, S), que genera un perfil de aversión al riesgo, conjugado simultáneamente con el deseo de incrementar el capital (Potencial, $P$ ), que corresponde con un perfil de propensión al riesgo; por el otro lado, hay un objetivo de alcanzar un estatus financiero determinado (Aspiración, $A$ ). Este trabajo fue ampliado junto con Gregg Oden en The role of aspiration level in risky choice (1999). Estos autores plantean que, a partir de una riqueza inicial $W_{0}$, pueden presentarse $n$ posibles niveles de riqueza al cabo del horizonte de tiempo considerado, cada uno asociado con una probabilidad $p_{i}$, de forma que la riqueza esperada se expresa como:

$$
E(W)=\sum_{i=1}^{n} p_{i} W_{i}=\sum_{i=1}^{n} D_{i}
$$

donde $D_{i}$ denota la probabilidad del aumento consecutivo de la riqueza en cada periodo. $\mathrm{Si}$ el inversionista es influenciado por la esperanza, tenderá a sobreestimar la probabilidad de los mejores escenarios y a subestimar la de los peores, situación en la que el exceso de optimismo lo llevará a buscar el Potencial. Por el contrario, si el inversionista es presa del temor, sobreestimará la probabilidad de obtener malos resultados y subestimará la de los buenos $\left(p_{n}<p_{1}\right)$ llevándolo a estructurar portafolios que le generen Seguridad. En otras palabras, si hay dos inversionistas que se enfrentan al mismo riesgo o "prospectiva", pero cada uno de ellos experimenta un nivel de miedo diferente, el más temeroso dará un mayor valor a la probabilidad de ocurrencia de hechos adversos, mientras que el menos temeroso le dará una menor probabilidad (Shefrin, 2010). De esta forma, se plantea una "función desacumulativa" $h(D)$ que refleja el peso de cada emoción, así: 


$$
h(D)=\delta h_{s}(D)+(1-\delta) h_{p}(D)
$$

Ya que $D$ es una función de distribución desacumulativa, la probabilidad asignada a la ocurrencia del hecho más desfavorable $x_{1}$ está dada por: Prob $\left\{x_{1}\right\}=h\left(D\left(x_{1}\right)\right)-h\left(D\left(x_{2}\right)\right)$. Como se afirmó, un inversionista temeroso ante un posible hecho perjudicial para su portafolio sobreestima su probabilidad de ocurrencia, llevando a que: $h\left(D\left(x_{1}\right)\right)-h\left(D\left(x_{2}\right)\right)$ $>D\left(x_{1}\right)-D\left(x_{2}\right)$.

En presencia de miedo se tiende a buscar la Seguridad (expresada en el subíndice $s$ ) y el valor esperado de la riqueza $E(W)$ es calculado con la función desacumulativa $h_{s}(D)=D_{s}{ }^{1+q}$, donde $q>0$ representa la estimación de $D$. Si hay optimismo hacia el futuro, el inversionista apuntará al Potencial (subíndice $p$ ) y el valor esperado de su riqueza estará dado por $h_{p}(D)=1-(1-D)^{1+q p}$. Como resultado, la función desacumulativa que combina las dos emociones se formula como: $h(D)=\lambda h_{s}(D)+(1-\lambda) h_{p}(D)$, donde $\lambda$ es la ponderación relativa de la seguridad y el potencial: un miedo extremo hace que $\lambda=1$, mientras un optimismo extremo lleva a que $\lambda=0$. En cualquier caso intermedio, habrá una combinación de miedo y esperanza $(0<\lambda<1)$ que determinará la composición del portafolio de inversión.

Bajo este enfoque, la Aspiración $(A)$ consiste en un valor o un rango de valores $\alpha$ al cual el inversionista desearía llegar; el riesgo de tal nivel de Aspiración está definido como la probabilidad $A=$ Prob $\{x \geq \alpha\}$ tal que el resultado aleatorio $x$ alcance o supere el nivel deseado $\alpha$. De esta forma, el inversionista tiene una función de utilidad objetivo dada por $V(S P, A)$, la cual es monótona estrictamente creciente en sus dos argumentos, de manera que puede estar dispuesto a renunciar a algo de Seguridad y Potencial $(S P)$ a cambio de obtener con mayor certeza su nivel de Aspiración $(A)$.

En años más recientes, el enfoque SP/A ha sido la base para desarrollar nuevos estudios y aplicaciones: por ejemplo, el impacto de la aspiración en la toma de decisiones fue analizado por Bruhin, Hehr-Duda y Epper (2009), así como por Diecidue y van de Ven (2008). Igualmente, Payne (2005) realizó una serie de experimentos mediante los cuales concluyó que, dada la inclusión de la variable Aspiración, el enfoque SP/A es más preciso para predecir la toma de decisiones que el modelo desarrollado por Kahneman y Tversky en la Teoría de la Prospectiva.

Se puede afirmar que el enfoque SP/A es una buena alternativa a la Teoría de la Prospectiva de Kahneman y Tversky, como análisis descriptivo de la toma de decisiones riesgosas. 
El modelo SP/A supone -de forma realista- que los inversionistas son motivados por la seguridad o por el potencial, de acuerdo con sus motivaciones personales, pero, a diferencia del enfoque tradicional en que un inversionista está en uno de dos polos opuestos (averso o propenso al riesgo), en este análisis los dos perfiles de riesgo están presentes en cada persona en diferentes niveles, los cuales reflejan cómo es su forma particular de percibir el riesgo en cada momento. Este modelo tiene bases psicológicas, como son la motivación y la atención, elementos básicos de la toma de decisiones financieras; particularmente, la aspiración es un punto de referencia que representa tres factores psicológicos que se desprenden del perfil del inversionista, como son la influencia de las alternativas estudiadas, la esperanza razonable y las influencias externas. En resumen, el enfoque SP/A brinda un marco analítico en el que, influenciados por el miedo y la esperanza, los inversionistas construyen portafolios con los que buscan asegurarse un nivel mínimo de riqueza $(S)$, al tiempo que tener la oportunidad de ir incrementando con el tiempo el valor de su capital $(P)$ y apostar a alcanzar un nivel aspiracional de riqueza $(A)$.

\subsection{Portafolios comportamentales (Behavioral portfolios)}

En el artículo Behavioral Portfolio Theory (2000), Hersh Shefrin y Meir Statman desarrollaron un enfoque comportamental descriptivo para la selección de portafolios, contrastándolo con el enfoque prescriptivo de Markowitz, concluyendo que la composición obtenida en cada uno de ellos así como las fronteras eficientes son diferentes, ya que mientras los agentes de la Teoría Moderna del Portafolio se basan en el análisis de media-varianza, los inversionistas de la Teoría Comportamental eligen la combinación de activos basados en la riqueza esperada, su necesidad de seguridad y su nivel aspiracional; factores previamente propuestos por Lopes (1987) y Kahneman y Tversky (1979). Otra diferencia radica en que los primeros eligen una combinación del activo libre de riesgo y del portafolio de mercado acorde con el CAPM, mientras los portafolios de los segundos se componen de una mezcla de bonos y “billetes de lotería” (Bourachnikova, 2002).

Shefrin y Statman usaron en su análisis el concepto comportamental descrito inicialmente por Thaler (1980) como "contabilidad mental", descrito en el apartado 1.2 de este artículo. Con base en tal concepto, presentaron dos versiones de su teoría: en una de ellas, suponen que los inversionistas tienen en cuenta la media, la varianza y la covarianza entre los activos, y con base en ellas construyen un solo portafolio, es decir, hacen una sola "cuenta mental" (Single Account: BPT/SA); en la otra, construyen simultáneamente diferentes portafolios, cada uno en una diferente "cuenta mental", pasando por alto la 
covarianza entre ellos (Multiple Accounts: BPT / MA). Ambos enfoques son analizados a continuación.

\subsubsection{Portafolio comportamental / Cuenta única (Behavioral Portfolio /Single Account)}

En este enfoque, Shefrin y Statman parten de suponer un inversionista con un horizonte de tiempo de un periodo, que en el momento inicial asigna su riqueza $W_{0}$ a la construcción de un portafolio, ignorando las relaciones entre los activos que lo componen, con el deseo de maximizar su consumo futuro, tratando de evitar que su riqueza llegue a ser menor que un determinado nivel aspiracional $A$; por lo tanto, adquiere una serie de pagos contingentes denotados $W_{1}, W_{2}, \ldots, W_{n^{\prime}}$ considerando una probabilidad dada de que ellos no alcancen el nivel de aspiración $P\{W \leq A\}$. Cada pago contingente es una variable dicotómica que toma el valor 1 si se cumple el estado $i$, o 0 si no se cumple (una unidad de consumo o ninguna al final del periodo), y tiene un precio $v_{i}$ en el estado $i$, con probabilidad de ocurrencia $p_{\mathrm{i}}$. Como resultado, el objetivo del inversionista será que al final del periodo logre maximizar $E_{h}(W)=\sum r_{i} W_{i}$ sujeto a su restricción presupuestaria dada por $\sum v_{i} W_{i} \geq W_{0}$ (no puede invertir en activos más del monto con que cuenta en el momento cero).

Suponga que el inversionista cuenta con $\$ 100$ para asignar a cuatro estados posibles, y tiene un nivel aspiracional de $\$ 90$, es decir, sabe que puede obtener una rentabilidad positiva, pero no tolerará una pérdida de más del $10 \%$ de su inversión. Los precios de los cuatros estados son respectivamente $\$ 14, \$ 19, \$ 28$ y $\$ 39$, cada uno de estos estados tiene una misma probabilidad del $25 \%$ de darle al inversionista su nivel aspiracional de $\$ 90$, y a su vez cada uno de ellos genera el mismo aporte en términos de unidades de consumo futuro (por cada unidad adquirida en cualquier estado, recibirá una unidad de consumo). ¿Cómo debería entonces conformar el portafolio para maximizar su consumo, contar con el nivel de aspiración determinado y cumplir con la restricción presupuestaria? En este enfoque no hay respuesta única, pues dependerá del perfil del inversionista, expresado en la probabilidad que desee definir de alcanzar el nivel aspiracional: por ejemplo, suponga que esta es una persona poco aversa al riesgo y por lo tanto decide que conformará un portafolio que le permita tener $A=90$ con $25 \%$ de probabilidad, caso en el que tomará sus $\$ 100$ y comprará solamente unidades del estado más barato (el 1), a un precio de $\$ 14$ cada una, obteniendo 100 / $14=7,14$ unidades de consumo, como se puede observar en la Tabla 1. 


\section{Tabla 1}

Portafolio comportamental - Baja aversión al riesgo

\begin{tabular}{ccccc}
\hline Estado $\mathrm{i}$ & Precio $\left(\mathrm{v}_{\mathrm{i}}\right)$ & Probabilidad $\mathrm{A}=90\left(\mathrm{p}_{\mathrm{i}}\right)$ & Unidades consumidas & Dinero invertido \\
\hline 1 & 14 & $25 \%$ & 7,14 & 100,00 \\
2 & 19 & $25 \%$ & 0 & 0 \\
3 & 28 & $25 \%$ & 0 & 0 \\
4 & 39 & $25 \%$ & 0 & 0 \\
\hline Total & & & 7,14 & 100,00 \\
\hline
\end{tabular}

Fuente: elaboración propia

Ahora bien, si fuera un inversionista bastante averso al riesgo, desearía obtener con $100 \%$ de probabilidad el nivel de aspiración, por lo que tendría que adquirir al menos una unidad de cada estado: la asignación óptima se encontraría comprando consecutivamente una unidad de los niveles más caros, y el dinero restante en cuantas unidades fuera posible del más barato; en este caso analizado, su portafolio estaría compuesto por una unidad de cada estado, dando como resultado cuatro unidades de consumo, como muestra la Tabla 2; este portafolio puede asimilarse a invertir en un activo libre de riesgo, pues garantiza la obtención del nivel aspiracional definido, aunque el consumo final es inferior al de un portafolio más arriesgado.

\section{Cuadro 2}

Portafolio Comportamental - Alta aversión al riesgo

\begin{tabular}{ccccc}
\hline Estado $\mathrm{i}$ & Precio $\left(\mathrm{v}_{\mathrm{i}}\right)$ & Probabilidad $\mathrm{A}=90\left(\mathrm{p}_{\mathrm{i}}\right)$ & Unidades consumidas & Dinero invertido \\
\hline 1 & 14 & $25 \%$ & 1 & 14 \\
2 & 19 & $25 \%$ & 1 & 19 \\
3 & 28 & $25 \%$ & 1 & 28 \\
4 & 39 & $25 \%$ & 1 & 39 \\
\hline Total & & & 4 & 100 \\
\hline
\end{tabular}

Fuente: elaboración propia

Shefrin (2008) propone la siguiente generalización: si todos los estados son igualmente probables, existe un estado crítico $i_{c}$ tal que el portafolio óptimo tiene la siguiente forma:

$$
\begin{gathered}
c_{i}=0 \text { para } i<i_{c} \\
c_{i}=A \text { para } i_{c} \leq i<n
\end{gathered}
$$




$$
c_{n}=\frac{\left(W-\sum_{i=1}^{n-1} v_{i} c_{i}\right)}{V_{n}}
$$

Donde $i_{c}$ es el menor entero para el cual $\sum_{i>i c} p_{i} \geq A$.

\subsubsection{Portafolio comportamental / Múltiples cuentas} (Behavioral portfolio/ Multiple accounts)

Bajo este enfoque, el inversionista se fija dos metas simultáneas, pero de forma separada (en dos cuentas mentales diferentes, sin tener en cuenta la correlación entre ellas): por un lado, desea evitar que su riqueza caiga por debajo de un nivel de aspiración, mientras por el otro se siente atraído por la posibilidad (mínima en la práctica) de ganar en una lotería que le permita enriquecerse (De Giorgi, 2011; Fong, 2014).

Nuevamente suponga que el inversionista tiene una riqueza inicial $W_{0}=\$ 100$, y evalúa cómo asignarla entre dos activos $X$ y $Y$, cuyos retornos siguen una distribución normal y tienen cero correlación. Esta persona se fija dos niveles de aspiración: uno mínimo $\left(A_{L}\right)$ de $\$ 90$, y uno alto $\left(A_{H}\right)$ de $\$ 150$, lo que se puede representar con dos funciones de utilidad diferentes $\left(U_{L}\right.$ y $U_{H^{\prime}}$ respectivamente); $W_{i}$ es el valor que puede tener la riqueza en cada cuenta mental al final del periodo, con una probabilidad $P_{i}$ de no obtener cada uno de los niveles mencionados de aspiración $A_{i}$. Shefrin y Statman plantean funciones de utilidad Cobb-Douglas, con parámetros $\gamma, \beta \in(0,1)$, de la siguiente manera:

$$
\begin{aligned}
& U_{L}=P_{L}^{1-\gamma} E_{h}\left(W_{L}\right)^{\gamma} \\
& U_{H}=P_{H}^{1-\beta} E_{h}\left(W_{H}\right)^{\beta}
\end{aligned}
$$

La utilidad total combina las dos anteriores funciones, con un peso $K_{d i}$ asignado a cada cuenta $\left(K_{d H}>K_{d L}\right)$, lo que resulta en:

$$
U=\left[1+K_{d H}\left(P_{H}^{1-\beta} E_{h}\left(W_{H}\right)^{\beta}\right)\right] K_{d L}\left[P_{L}^{1-\gamma} E_{h}\left(W_{L}\right)^{\gamma}\right]
$$


Puede observarse cómo el inversionista no tendrá ninguna utilidad en caso de no alcanzar su nivel mínimo de aspiración: si $P_{L}^{1-\gamma} E_{h}\left(W_{L}\right)^{\gamma}=0$ entonces $U=0$. Ahora, de no obtener el nivel de aspiración alto (por ejemplo, si no gana la lotería), su utilidad total dependerá solamente del nivel aspiracional mínimo: si $P_{H}^{1-\beta} E_{h}\left(W_{H}\right)^{\beta}=0$ entonces $U=K_{d L}\left[P_{L}^{1-\gamma} E_{h}\left(W_{L}\right)^{\gamma}\right]$. El portafolio óptimo de cada nivel de aspiración corresponde a una solución de esquina, pues si el inversionista desea obtener $A_{L}$ con toda certeza, adquirirá solamente activos libres de riesgo, mientras que si desea tener la posibilidad de obtener $A_{H^{\prime}}$ asignará todo su dinero a apostar con billetes de lotería (Barberis y Huang, 2008; Barberis y Xiong, 2012); dado que ambas aspiraciones están ocurriendo mentalmente de forma simultánea, Shefrin (2008) denomina a este inversionista como "bipolar", pues se va a los extremos en la conformación de su portafolio, adquiriendo una combinación de activo libre de riesgo y billetes de lotería.

Como se observa, Shefrin y Statman se apartan del supuesto básico de que el inversionista elige su portafolio con base en la media y la varianza de los activos, concentrándose en el valor esperado de su riqueza, el deseo de seguridad y la aspiración de alcanzar unos retornos potenciales, propios de diferentes niveles aspiracionales. Un importante aporte es la introducción de las cuentas mentales, por medio de las cuales el inversionista estructura diversos portafolios, cada uno orientado a cumplir un objetivo específico planteado para cada nivel de su "pirámide escalonada" de fines, y acorde con una tolerancia diferente al riesgo. El enfoque del portafolio comportamental resulta bastante útil para asesores financieros, particularmente en el área de los fondos de pensiones, pues es consistente con la tendencia del ser humano a llevar diferentes cuentas mentales; de esta manera, es posible diseñar sub-portafolios para los clientes, de manera que pueda simultáneamente contar con unos fondos seguros para su retiro, pero al tiempo esté en la posibilidad de acceder a retornos provenientes de inversiones especulativas. Tal posibilidad no es considerada por el modelo de Markowitz, en el que el inversionista es siempre averso al riesgo y, por tanto, no contempla la posibilidad de asignar recursos a activos similares a una lotería.

\subsection{Portafolio maslowiano (Maslowian portfolio)}

En 2009, Philippe De Brouwer publicó el artículo Maslowian Portfolio Theory, an alternative formulation of the Behavioral Portfolio Theory, en el que describió la similitud entre la teoría psicológica de la jerarquía de las necesidades formulada por Abraham H. MasLow (1943), y la Teoría Comportamental del Portafolio de Shefrin y Statman (2000). La Pirámide de Maslow plantea una escala de necesidades del ser humano de la siguiente 
manera: en la base se encuentra la fisiología, y a medida que asciende están la seguridad, la afiliación, el reconocimiento y finalmente la autorrealización. La analogía que hace De Brouwer consiste en que, de la misma forma, las personas tienen necesidades ordenadas en escalones en términos de su vida financiera, las cuales se van satisfaciendo gradualmente, en un proceso de decisión influenciado por factores no monetarios (psicológicos, emocionales, de entorno), de manera que hay un portafolio de inversión "óptimo" para cada uno de esos escalones, así:

1. En la base se encuentra el nivel fisiológico, relacionado con la obligación de contar con recursos para satisfacer las necesidades básicas (alimento, vestido, transporte, servicios públicos, etc.), de manera que se requiere principalmente dinero en efectivo u otros medios de pago líquidos. Una persona con bajos ingresos se situará en este primer escalón y todo su "portafolio" se encontrará en efectivo (para minimizar el riesgo y maximizar la liquidez); a medida que el ingreso aumenta, el porcentaje de efectivo en el portafolio disminuye.

2. A continuación está la necesidad de seguridad, es decir, la preocupación de contar con activos que generen flujo de caja y permitan satisfacer al menos las necesidades fundamentales futuras. Entran aquí al portafolio activos como dinero en un fondo de retiro, seguros básicos y bienes inmuebles; en este sentido, el portafolio óptimo es uno similar al del enfoque "Primero la Seguridad".

3. En el tercer nivel se sitúa la necesidad de afiliación, llamada también por De Brouwer como necesidad de amor, relacionada con el deseo de lograr mejores condiciones presentes y futuras de vida para el grupo familiar; es así como el portafolio de inversiones incluye en este nivel cuentas de ahorros, recursos en fondos de inversión y pólizas de largo plazo como seguros educativos. El portafolio resultante es del tipo SP/A.

4. A continuación se encuentra el nivel de reconocimiento, cuando se han cubierto las necesidades de los escalones básicos, se incrementa en el portafolio la tenencia de activos financieros más personalizados, al tiempo que los recursos financieros excedentes empiezan a ser usados en la adquisición de bienes y servicios de lujo, a través de los cuales no solo se aumente el bienestar sino también la reputación y el prestigio social. Los portafolios óptimos de este nivel pueden bien ser hallados por el método media-varianza de Markowitz.

5. En la parte más alta de la pirámide está la realización personal, que en términos financieros significa que el ahora inversionista sofisticado no solamente se preocupa de su propia riqueza, sino también de causas humanitarias, de manera que su portafolio se compone de inversiones altamente especializadas, al tiempo que 
de recursos para causas filantrópicas. El enfoque de Markowitz puede emplearse para hallar el portafolio de este nivel, junto con decisiones más arriesgadas como las apuestas.

Siguiendo a Majewski $(2010,2014)$, este problema puede ser formulado de la siguiente manera: el inversionista adquiere una serie de $n$ activos $x_{i j}$, cada uno en una proporción $w_{j^{\prime}}$ conformado un número $k$ de sub-portafolios $(s)$ dentro del conjunto del total de su inversión $(p)$, pasando por alto la posible correlación entre ellos, de manera que el retorno total de su portafolio $\left(\mu_{p}\right)$ será el promedio ponderado de los retornos de las rentabilidades obtenidas en cada escalón o compartimento $\left(\mu_{s j}\right)$, así:

$$
\begin{aligned}
& \mu_{s j}=\sum_{i=1}^{n} \mu_{i j} w_{i j} \\
& \mu_{p}=\sum_{j=1}^{k} \mu_{s j} w_{j}
\end{aligned}
$$

El objetivo de este inversionista será obtener la mejor rentabilidad posible de cada compartimento, es decir, maximizar $\mu_{s j^{\prime}}$ sujeto a $\sum x_{i j}=1$. Si las ventas en corto no están permitidas para todos los activos, otra restricción es que $x_{i j} \geq 0$, pero si en algún activo las ventas en corto no son posibles, es necesario agregar para ellos la restricción $x_{i j} \in R$. Ahora bien, el hecho de que el inversionista pase por alto las correlaciones entre los activos no significa que ella no tenga influencia sobre los resultados obtenidos, de forma que el riesgo de su inversión -medido por la desviación estándar de los retornos $\left(\sigma_{p j}\right)$ - puede expresarse como:

$$
\sigma_{p j}=\sqrt{X^{T} D X}
$$

donde $X$ es el vector de activos incluidos en cada sub-portafolio y $D$ la matriz de covarianzas entre ellos. El inversionista, al tiempo que trata de maximizar $\mu_{s j}$ para los activos situados en la parte superior de la pirámide, buscará evitar en cuanto sea posible el riesgo en los escalones inferiores de la misma, es decir, minimizar $\sigma_{p j}$ ambos procesos sujetos a las restricciones mencionadas. Sin embargo, Majewski propone que tal minimización también se refiere a la necesidad de liquidez para estos niveles inferiores de la pirámide, por lo que se puede agregar otra función objetivo independiente $L_{p j^{\prime}}$ que mide la liquidez de cada j-ésimo sub-portafolio: 


$$
L_{p j}=V_{p j} / V_{g}
$$

donde $V_{p j}$ y $V_{g}$ son, respectivamente, el volumen transado en el portafolio del inversionista, y el volumen total del mercado del cual provienen los activos en él incluidos. En este caso, el objetivo del inversionista puede ser maximizar la función de liquidez $L_{p j}$ sujeto a las mismas tres restricciones antes mencionadas: $\sum x_{i j}=1, x_{i j} \geq 0$ para activos que no permitan ventas en corto, y si ellas son posibles, $x_{i j} \in R$.

Enfoques como el de De Brower permiten ver que en la actualidad el análisis de las decisiones de portafolio no se limita a la búsqueda de una asignación óptima a partir de una función objetivo dada, sino que incluye el proceso que toma lugar durante la decisión de inversión: de hecho, en este enfoque lo importante no es la estructura final del portafolio, sino el mecanismo con el que se estructura. Muchos agentes de los mercados financieros no solamente buscan maximizar sus retornos, sujetos a una serie de restricciones, sino que tienen en cuenta aspectos que la literatura tradicional no había considerado hasta el momento, como los relacionados con la responsabilidad social y los fines humanitarios.

En resumen, De Brower fusiona en su planteamiento la teoría psicológica de Maslow con la teoría comportamental de Shefrin y Statman, permitiendo comprender que no hay un único portafolio óptimo para el inversionista, sino, más bien, diversos portafolios que responden a objetivos ordenados en escalones, los que se van generando a lo largo de su vida financiera, respondiendo a necesidades e intereses cambiantes, de forma que simultáneamente tiende a minimizar el riesgo en los escalones inferiores (correspondientes a necesidades básicas, para las cuales requiere dinero en efectivo), mientras desea maximizar el retorno en los escalones superiores (relacionados con inversiones sofisticadas y causas filantrópicas).

\section{Conclusiones}

La Teoría Moderna del Portafolio parte del supuesto paradigmático de la "racionalidad" de los agentes, el cual implica la construcción de modelos caracterizados por la optimización matemática en un marco de perfecta previsión, perfecto uso de la información y ausencia de elementos como las emociones, las influencias del entorno o los errores cognitivos, elementos que caracterizan a un ser humano real y que, indudablemente, influyen en sus decisiones financieras. 
El modelo media-varianza para la selección de carteras de inversión y el Modelo de Valoración de Activos Financieros (CAPM), fundamentos de la teoría y la práctica financieras tradicionales, tienen un carácter prescriptivo, más que descriptivo, es decir, muestran cómo agentes híper-racionales deberían tomar decisiones en el marco de un mercado de valores eficiente, más que explicar el comportamiento observado de los inversionistas, que son personas con capacidades limitadas, en cuyas elecciones presentan sesgos sistemáticos, producidos por aspectos emocionales, psicológicos y por la influencia del entorno.

A partir de las teorías de corte comportamental presentadas -en las que se incluyen factores no contemplados por el enfoque tradicional, como el miedo, las aspiraciones, las cuentas mentales, la necesidad de afiliación, el deseo de realización personal- es posible generar modelos financieros de corte descriptivo, que permitan comprender más acertadamente la toma de decisiones que en la práctica realizan las personas. A futuro, tales modelos deberán incluir aspectos emocionales y psicológicos como la culpa, la ira, el orgullo, la alegría, la tristeza, el amor, la desesperación, sin que eso implique renunciar a la rigurosidad cuantitativa; esto a su vez quiere decir que los enfoques tradicional y comportamental en Finanzas no deben ser vistos como sustitutos, sino como complementarios. Para tal fin, es necesario el trabajo conjunto de disciplinas como la economía, la administración, la psicología, la sociología y las neurociencias, entre otras. De igual manera, la influencia de los factores externos en las decisiones de inversión debe ser estudiada más profundamente, incluyendo el impacto de los factores del entorno, el grado de desarrollo de los mercados, la cultura, la normatividad, etc.

La investigación en el campo de las Finanzas del Comportamiento debe orientarse a la construcción de teorías y modelos en los que el grado de racionalidad de los agentes estudiados no sea siempre perfecto, sino que dependa, entre otros factores, del contexto analizado; de hecho, una nueva generación de modelos financieros debe suponer la coexistencia de inversionistas racionales y "cuasi racionales", quienes no manejan perfectamente la información, no resuelven los problemas de forma correcta en el primer intento, cometen errores, e incluso reinciden en ellos, a veces sistemáticamente, todo esto en el marco de mercados ineficientes. En este mismo orden de ideas, diferentes tipos de decisiones de inversión requieren de diferentes modelos que las expliquen: el proceso de selección y gestión del portafolio para la jubilación -un difícil problema con pocas oportunidades de aprendizaje- es claramente distinto del modelo de portafolio empleado por una firma de corredores dedicada a la negociación intradiaria de acciones. 
A la fecha, es posible afirmar que las Finanzas del Comportamiento están en proceso de construcción como una rama sólida de las finanzas; en particular, "el estudio sobre la selección comportamental de portafolios está aún en su infancia" (Zhang et al., 2011, p. 256), en particular en áreas como la formalización cuantitativa en modelos de tiempo continuo.

La inclusión de elementos comportamentales - para algunos el nuevo paradigma en Finanzas (Jaye, 2014)- permite contar con una teoría más adecuada para la comprensión del comportamiento observado de los inversionistas, y su aplicación podrá ayudar a que ellos tomen mejores decisiones, al tiempo que contribuirá a que las instituciones financieras puedan en la práctica realizar procesos de asesoría más adecuados, que respondan con mayor precisión a las necesidades de los clientes inversionistas, con alternativas que se ajusten verdaderamente a sus expectativas personales e intereses específicos.

\section{Referencias}

Ajzen, I. (1991). The theory of planned behavior. Organizational Behavior and Human Decision Processes, 50, 179-211.

Akerlof, G. A., and Dickens, W. T. (1982). The economic consequences of cognitive dissonance. American Economic Review, 72 (3), 307-319.

Arcos, M., Benavides, J., and Breggrun, L. (2010). Optimal portfolio allocation for Latin American stock indices. Cuadernos de Administración, 23 (40), 191-214.

Auðunsdóttir, E. (2011). Modern portfolio theory: Does it work? Tesis doctoral. University of Iceland, Reikiavik, Islandia. Recuperado el 22 de julio de 2015 de: http://hdl.handle. net/1946/7233

Baker, K. H., and Nofsinger, J. R. (2002). Psychological biases of investors. Financial Services Review, 11 (2), 97-116.

Baker, K. H., and Ricciardi, V. (2014). Investor behavior: The psychology of financial planning and investing ( $1^{\text {st }}$ ed.). Hoboken: John Wiley \& Sons.

Baker, M. P., and Wurgler, J. (2013). Behavioral corporate finance: An updated survey. In: G. M. Constantinides, M. Harris, and R. M. Stulz (Eds.), Handbook of the Economics of Finance, Vol. 2A (pp. 357-424). Amsterdam: Elsevier Press.

Barber, B. M., and Odean, T. (2000). Trading is hazardous to your wealth: The Common stock investment performance of individual investors. The Journal of Finance, 55 (2), 773-806.

Barber, B. M., and Odean, T. (2001). Boys will be boys: Gender, overconfidence, and common stock investment. The Quarterly Journal of Economics, 116 (1), 261-292. 
Barberis, N., and Huang, M. (2008). Stocks as lotteries: The implications of probability weighting for security prices. American Economic Review, 98 (5), 2066-2100.

Barberis, N., and Thaler, R. (2003). A survey of behavioral finance. In: G. M. Constantinides, M. Harris, and R. M. Stulz (Eds.), Handbook of the Economics of Finance (pp. 1053-1123). Amsterdam: Elsevier.

Barberis, N., and Xiong, M. (2012). Realization utility. Journal of Financial Economics, 104 (2012), 251-271.

Bell, D. (1982). Regret in decision making under uncertainty. Operations Research, 30 (5), 961-981.

Ben Mohamed, E., and Abdelfatteh, B. (2012). Portfolio selection between rational and behavioral theories emergent markets case. Management Science Letters, 2 (4), 1219-1232.

Beyer, S., and Bowden E. M. (1997). Gender differences in self-perceptions: Convergent evidence from three measures of accuracy and bias. Personality and Social Psychology Bulletin, 23 (2), 157-172.

Bishop, R. C. (2007). The Philosophy of the Social Sciences. London: Continuum International Publishing Group.

Black, F., and Litterman, R. (1991). Asset allocation: Combining investors views with market equilibrium. The Journal of Fixed Income, 1 (2), 7-18.

Bourachnikova, 0. (2002). Weighting function in the Behavioral Portfolio Theory. Département d'Economie Appliquée de l'Université Libre de Bruxelles, Working Paper 07-07 RS. Recuperado el 22 de julio de 2015 de: http://dev.ulb.ac.be/dulbea/fr/workingpaper.php

Brennan, T. J., and Lo, A. W. (2011). An evolutionary model of bounded rationality and intelligence. PLOS ONE, 7 (11), 1-8.

Bruhin, A., Hehr-Duda, H., and Epper T. (2009). Uncertainty breeds decreasing impatience: The role of risk preferences in time discounting. University of Zurich, Working Paper No. 412. Recuperado el 22 de julio de 2015 de: http://ssrn.com/abstract=1416007

Chandra, A. (2008). Decision making in the stock market: Incorporating psychology with finance. National Conference on Forecasting Financial Markets of India, 2008. Recuperado el 22 de julio de 2015 de: http://ssrn.com/abstract=1501721

Cooke, R., and Sheeran, P. (2004). Moderation of cognition-intention and cognition behaviour relations: A meta-analysis of properties of variables from the theory of planned behaviour. British Journal of Social Psychology, 43 (2), 159-186.

Curtis, G. (2004). Modern portfolio theory and behavioral finance. The Journal of Wealth Management, 7 (2), 16-22.

Daniel, K., Hirshleifer, D., and Teoh, S. (2002). Investor psychology in capital markets: Evidence and policy implications. Journal of Monetary Economics, 49 (2002), 139-209.

De Bondt, W. (1998). A portrait of the individual investor. European Economic Review, 42, 831-844. 
De Bondt, W., and Thaler, R. H. (1985). Does the stock market overreact? The Journal of Finance, 40 (3), 793-805.

De Brouwer, P. J. S. (2009). Maslowian portfolio Theory: An alternative formulation of the Behavioural Portfolio Theory. Journal of Asset Management, 9 (6), 359-365.

De Giorgi, E. (2011). A behavioral explanation of the asset allocation puzzle. Investment Management and Financial Innovations 8 (4), 36-44.

Diecidue, E., and Van de Ven, J. (2008). Aspiration level, probability of success and failure, and expected utility. International Economic Review, 49 (2), 683-700.

Dowling, M., and Lucey, B. (2005). The role of feelings in investor decision-making. Journal of Economic Surveys, 19 (2), 211-237.

Dubova, I. (2005). La validación y aplicabilidad de la Teoría de Portafolio en el caso colombiano. Cuadernos de Administración, 18 (30), 241-279.

Eagles, J. (1994). The relationship between mood and daily hours of sunlight in rapid cycling bipolar illness. Biological Psychiatry, 36 (6), 422-424.

Edmans, A., García, D., and Norli, 0. (2007). Sports sentiment and stock returns. The Journal of Finance, 62 (4), 1967-1998.

Fairchild, R., Muradoglu, G., and Chena, X. (2014). Between fear and hope: Optimal portfolio choice in a model combining expected utility and safety first preferences. Working Paper. University of Bath / Queen Mary University of London. Recuperado el 16 de julio de 2015 de: http://www.busman.qmul.ac.uk/research/Behavioural\%20Finance\%20Working\%20Group/132889.pdf

Fan, Y., Ying, S., Wang, B., and Wei, Y. (2009). The effect of investor psychology on the complexity of stock market: An analysis based on cellular automaton model. Computers \& Industrial Engineering, 56 (1), 63-69.

Fazio, R. H. (1986). How do attitude guide behavior? In: R. Sorrentino, and E. T. Higgins (Eds.), Handbook of motivation and cognition: Foundation of social behavior (pp. 204-243). New York: Guilford Press.

Fischhoff, B. (1982). For those condemned to study the past: Heuristics and biases in hindsight. In: D. Kahneman, P. Slovic, and A. Tversky (Eds.), Judgment under Uncertainty: Heuristics and Biases (pp. 80-98). Cambridge: Cambridge University Press.

Fishbein, M., and Ajzen, I. (1975). Belief, attitude, intention and behavior: An introduction to theory and research. Reading: Addison-Wesley.

Fong, W. M. (2014). The lottery mindset: Investors, gambling and the stock market. Basingstoke: Palgrave Macmillan.

Forgas, J. (1995). Mood and judgment: The affect illusion model (Aim). Psychological Bulletin, 117 (1), 39-66. 
Frijns, B.; Koellen, E., and Lehnert, T. (2008). On the determinants of portfolio choice. Journal of Economic Behavior \& Organization, 66 (2), 373-386.

González, M. y Nave, J. M. (2014). ¿Los índices de mercado son carteras eficientes? El caso español del IBEX-35. Cuadernos de Administración, 27 (48), 183-226.

Haley, M. R.; Paarsch, H. J., and Whiteman, C. H. (2013). Smoothed safety first and the holding of assets. Quantitative Finance, 13 (2), 167-176.

Hirshleifer, D. (2001). Investor psychology and Asset Pricing. Journal of Finance, 56 (5), 1533 1597.

Jaye, N. (2014). The next paradigm. CFA Institute Magazine, 25 (5), 28-31.

Johnson, E., and Tversky, A. (1983). Affect, generalization and perception of risk. Journal of Personality and Social Psychology, 45 (1), 20-31.

Jolls, C., Sunstein, C. R., and Thaler, R. H. (1998). A behavioral approach to law and economics. Stanford Law Review, 50 (5), 1471-1550.

Kahneman, D., and Tversky, A. (1979). Prospect theory: An analysis of decision under risk. Econometrica, 47 (2), 263-291.

Kahneman, D., and Tversky, A. (1981). The framing of decisions and the psychology of choice. Science, 211 (4481), 453-458.

Kahneman, D., and Tversky, A. (1986). Rational choice and the framing of decisions. The Journal of Business, 59 (4), S251-S278.

Kamstra, M. J., Kramer, L. A., and Levi, M. D. (2003). Winter Blues: A SAD Stock Market Cycle. American Economic Review, 93 (1), 1257-1263.

Kreps, D. M. (2012). Microeconomic Foundations I: Choice and Competitive Markets. Princeton: Princeton University Press.

Kuhnen, C. M., and Knutson, B. (2011). The influence of affect on beliefs, preferences, and financial decisions analysis. Journal of Financial and Quantitative Analysis, 46 (3), 605-626.

Lakonishok, J., Shleifer, A., and Vishny, R. W. (1994). Contrarian investment, extrapolation, and risk. Journal of Finance, 49 (5), 1541-1578.

Langer, E., and Roth, J. (1975). Heads I win tails it's chance: The illusion of control as a function. Journal of Personality and Social Psychology, 32 (6), 951-955.

Levy, H., and Levy, M. (2009). The safety first expected utility model: Experimental evidence and economic implications. Journal of Banking \& Finance, 33 (8), 1494-1506.

Lintner, J. (1965). The valuation of risk assets and the selection of risky investments in stock portfolios and capital budgets. The Review of Economics and Statistics, 47 (1), 13-37.

Lo, A. W. (2005). Reconciling efficient markets with behavioral finance: The adaptive markets hypothesis. Journal of Investment Consulting, 7 (2), 21-44.

Loewenstein, G., Hsee, C., Weber, E., and Welsh, N. (2001). Risk as feelings. Psychological Bulletin, 127 (2), 267-286. 
Lopes, L. (1987). Between hope and fear: The psychology of risk. Advances in Experimental Social Psychology, 20, 255-295.

Lopes, L., and Oden, G. (1999). The role of aspiration level in risk choice: A comparison of Cumulative Prospect Theory and SP/A Theory. Journal of Mathematical Psychology, 43 (2), 286-313.

Majewski, S. M. (2010). Methodological aspects of behavioural portfolio with multitasking. Folia Oeconomica Stetinensia, 9 (1), 24-33.

Majewski, S. M. (2014). The Maslowian Portfolio Theory Versus the Pyramid Portfolio. Folia Oeconomica Stetinensia, 14 (1), 91-101.

Malevergne, Y., and Sornette, D. (2005). Higher-moment portfolio theory. The Journal of Portfolio Management, 31 (4), 49-55.

Markowitz, H. M. (1952). Portfolio Selection. The Journal of Finance, 7 (1), 77-91.

Markowitz, H. M. (1959). Portfolio Selection: Efficient Diversification of Investments (2 $\left.{ }^{\text {nd }} \mathrm{Ed}.\right)$. Malden: Wiley / Yale University Press.

Mas-Colell, A., Whinston, M. D., and Green, J. R. (1995). Microeconomic Theory. 0xford: 0xford University Press.

Maslow, A. H. (1943). A theory of human motivation. Psychological Review, 50 (4), 370-396.

Mayfield, C., Perdue, G., and Wooten, K. (2008). Investment management and personality type. Financial Services Review, 17 (3), 219-236.

Merton, R. C. (1969). Lifetime portfolio selection under uncertainty: The continuous-time case. Review of Economics and Statistics, 51 (3), 247-257.

Miller, D., and Ross, M. (1975). Self-serving bias in attribution of causality: Fact or fiction? Psychological Bulletin, 82 (2), 213-225.

Mossin, J. (1966). Equilibrium in a Capital Asset Market. Econometrica, 34 (4), 768-783.

Nawrocki, D., and Viole, F. (2014). Behavioral finance in financial market theory, utility theory, portfolio theory and the necessary statistics: A review. Journal of Behavioral and Experimental Finance, 2 (2014), 10-17.

Nofsinger, J. R. (2001). Investment Madness. How Psychology Affects Your Investing. London: Prentice Hall.

Nofsinger, J. R. (2002). Do optimists make the best investors? Corporate Finance Review, 6 (4), 11-17.

Nofsinger, J. R. (2012). The Psychology of Investing ( $5^{\text {th }}$ ed.). New York: Pearson.

Nofsinger, J. R., and Sias, R. W. (1999). Herding and feedback trading by institutional and individual investors. Journal of Finance, 54 (6), 2263-2295.

Olsen, R. (1997). Investment Risk: The experts perspective. Financial Analyst Journal, 53 (2), 62-66. 
Pascual-Ezama, D., Gil-Gómez de Liaño, B., and Scandroglio, B. (2015). The role of emotions arising from instability in investment behaviour / El papel de la carga emocional de la inestabilidad en el comportamiento de inversión. Estudios de Psicología, 36 (3), 656-682.

Pascual-Ezama, D., Pavonni, M., y Gil-Gómez de Liaño, B. (2010). Inversiones financieras y comportamiento del inversor: Behavioral Finance. Análisis Financiero Internacional, 140, 17-29.

Payne, J. W. (2005). It is whether you win or lose: The importance of the overall probabilities of winning or losing in risky choice. Journal of Risk and Uncertainty, 30 (1), 5-19.

Pyle, D. H., and Turnovsky, S. J. (1970). Safety-first expected utility maximization in mean-standard deviation portfolio analysis. Review of Economics and Statistics, 52, 75-81.

Rabin, M. (1998). Psychology and Economics. Journal of Economic Literature, 36 (1), 11-46.

Richards, T. (2014). Investing Psychology: The Effects of Behavioral Finance on Investment Choice and Bias. Hoboken: John Wiley \& Sons.

Roy, A. D. (1952). Safety first and the holding of assets. Econometrica, 20 (3), 431-450.

Russell, T., and Thaler, R. H. (1985). The relevance of quasi rationality in competitive markets. The American Economic Review, 75 (5), 1071-1082.

Samuelson, P. (1969). Lifetime portfolio selection by dynamic stochastic programming. Review of Economics and Statistics, 51 (3), 239-246.

Sapienza, P., and Zingales, L. (2013). Economic experts versus average Americans. American Economic Review, 103 (3), 636-642.

Schulmerich, M., Leporcher, Y. M., and Eu, C. H. (2015). Applied Asset and Risk Management: A Guide to Modern Portfolio Management and Behavior-Driven Markets. Berlin: Springer.

Sharpe, W. (1964). Capital asset prices: A theory of market equilibrium under conditions of risk. The Journal of Finance, 19 (3), 425-442.

Shefrin, H. (2007). Beyond Greed and Fear: Understanding Behavioral Finance and the Psychology of Investing. New York: Oxford University Press.

Shefrin, H. (2008). A Behavioral Approach to Asset Pricing. Burlington: Academic Press.

Shefrin, H. (2010). Behavioralizing Finance. Foundations and Trends in Finance 4. Hanover: Now Publishers Inc.

Shefrin, H., and Statman, M. (2000). Behavioral Portfolio Theory. The Journal of Finance and Quantitative Analysis, 35 (2), 127-151.

Shiller, R. J. (1990). Market Volatility and Investor behavior. American Economic Review, 80 (2), 58-62.

Shiller, R. J. (2003). Finance, Optimization, and the Irreducibly Irrational Component of Human Behavior. In: G. M. Constantinides, M. Harris, and R. M. Stulz (Eds.), The Handbook of the Economics of Finance (pp. 1125-1128). Amsterdam: Elsevier.

Sias, R. W. (2004). Institutional Herding. The Review of Financial Studies, 17 (1), 165-206.

Simon, H. A. (1993). Altruism and Economics. The American Economic Review, 83 (2), 156-161. 
Stael von Holstein, C. A. S. (1972). Probability forecasting: An experiment related to the stock market. Organizational Behavior and Human Performance, 8 (1), 139-158.

Statman, M. (2002). How much diversification is enough? Santa Clara University, Working Paper. Recuperado el 14 de julio de 2015 de: https://ssrn.com/abstract=365241.

Statman, M. (2014). Behavioral finance: Finance with normal people. Borsa Istanbul Review, 14 (2), 65-73.

Swedish Academy (2015), All Prizes in Economic Sciences. Recuperado el 22 de julio de 2015 de: http://www.nobelprize.org/nobel_prizes/economic-sciences/laureates/

Szyszka, A. (2013). Behavioral Finance and Capital Markets: How Psychology Influences Investors and Corporations. New York: Palgrave Macmillan.

Taffler, R. J., and Tuckett, D. A. (2010). Emotional finance: The role of the unconscious in financial decisions. In: H. K. Baker, and J. R. Nofsinger (eds.), Behavioral Finance. Investors, Corporations, and Markets (pp. 95-112). Hoboken: John Wiley \& Sons Inc.

Taylor, S., and Brown, J. (1988). Illusion and well-being: A social psychological perspective on mental health. Psychological Bulletin, 103 (2), 193-210.

Thaler, R. H. (1980). Toward a positive theory of consumer choice. Journal of Economic Behavior \& Organization, 1 (1), 39-60.

Thaler, R. H. (1985). Mental accounting and consumer choice. Marketing Science, 4 (3), 199-214.

Thaler, R. H. (1990). Savings, fungibility, and mental accounts. Journal of Economic Perspective, 4 (1), 193-205.

Thaler, R. H. (1999). The end of behavioral finance. Financial Analyst Journal, 55 (6), 12-17.

Thaler, R. H. (2000). From Homo Economicus to Homo Sapiens. Journal of Economic Perspectives, 14 (1), 133-141.

Tversky, A., and Kahneman, D. (1974). Judgment under uncertainty: Heuristics and biases. Science, 185 (4157), 1124-1131.

Tversky, A., and Kahneman, D. (1992). Advances in prospect theory: Cumulative representation of uncertainty. Journal of Risk and Uncertainty, 5 (4), 297-323.

Wright, W., and Bower, G. (1992). Mood effects on subjective probability assessment. Organizational Behavior and Human Decision Processes, 52 (2), 276-291.

Yuan, K., Zheng, L., and Zhu, Q. (2006). Are investors moonstruck? Lunar phases and stock returns. The Journal of Empirical Finance, 13 (1), 1-23.

Zhang, S., Jin, H., and Zhou, X. (2011). Behavioral portfolio selection with loss control. Acta Mathematica Sinica, English Series, 27 (2), 255-274. 
